\title{
Small Cell Discovery Technology Research and Analysis
}

\author{
Youqi Shen*, Guiyong Li and Kuan Wu \\ College of Communication and Information Engineering, Chongqing University of \\ Posts and Telecommunications, Chongqing 400065, China \\ syqsnail1128@126.com
}

\begin{abstract}
Small cell technology is an important emerging technology in LTE. It is a necessary deployment method for service hotspot area, enterprise users and indoor enhanced coverage. It is mainly used to enhance network capacity and optimize network deployment. Based on the research of LTE-A system standard and OFDM technology, combined with the characteristics of small cell. This paper analyzes the present research status of small cell discovery technology. At last, the conclusion and prospect are given.
\end{abstract}

Keywords: Small cell; interference; synchronization signal; discovery signal

\section{Introduction}

With the increasing capacity of LTE networks, mobile operators are worried about the growing demand for data traffic, small cell [1] is playing an increasingly important role in this area, so more small cells are used to cover blind spots and share data traffic pressure. Small cell enables operators to offer users higher data rates and lower cost services. Small cell intensive deployment is one of to improve system capacity means, 3GPP R12 carried out for small cell enhancement technology standardization work, including the introduction of high-order modulation (256QAM). According to traffic and interference cell switch, cell fast discovery and optimization of different operators between small cell intensive deployments of synchronization mechanisms. However, based on the traditional LTE cell search method, which is based on the PSS/SSS mechanism applied to small cell discovery, which there will be a series of problems.

\section{Cell Search and Discovery}

The cell search procedure is a process in which the UE acquires the time and frequency synchronization of the serving cell and detects the cell ID of the physical layer. According to the cell search process, the cell search is divided into an initial cell search and a noninitial cell search [2]. The initial cell search is the cell search after the user power on, the UE will find a suitable base station for itself to receive the message of the serving base station. Non-initial cell search refers to the UE is always in a connected state, periodically search for the presence of cells surrounding, the search to prepare for cell reselection and handover.

The discovery of small cell belongs to the non-initial cell search. Therefore, the noninitial cell search is simpler than the initial cell search. Because the non-initial cell search UE has already acquired downlink synchronization, but in order to reduce the complexity of the UE search service cell, the same method is used for the non-initial cell search and initial cell search.

UE can quickly and effectively discover small cell, which can reduce the network load of macro base stations, optimize network deployment and network load balancing, improve the effective operation of CoMP/e-ICIC and can effectively promote small cell 
shutdown technology research, also can enhance the mobile robustness of the small cell layer.

Fast turn-off and semi-static shut-down mechanism is the main problem of Small Cell shutdown technology. Semi-static shutdown mechanism The main research content is the Small Cell needs to be based on the user's departure and arrival, the end of the call group and arrival, business load reduction and increase and other factors to determine the Small Cell switch. According to the already existing LTE signaling flow, the semi-static shutdown mechanism can be turned off for hundreds of milliseconds or even a few seconds. In order to achieve fast sub-frame level LTE sub-mechanism, 3GPP R12 version of the switching cycle for the millisecond level of fast shutdown mechanism were discussed accordingly. Because the current LTE technology standard can not meet the small cell fast shutdown mechanism, 3GPP follow-up also need to continue through the convening of the relevant standard meeting to prove the feasibility of the program and to bring the system performance gain assessment.

\section{Discover the Mechanisms of Small Cell}

Small Cell discovery technology is the process by which a UE detects small cells and measures its RSRP / RSRQ. UE wants to access to the LTE network, will be through the cell search, access to the target cell system information and random access procedures. (Including symbol and frequency synchronization); get the system frame timing, that is, the starting position of the downlink frame; and finally determine the physical layer cell ID. Finally, the main purpose of this paper is as follows: 1 . In the context of intensive deployment of Small Cell, the current standard does not specify the number of small cells found, but most companies believe that the number of small cells found at least three.

In order to better support UE mobility, it is necessary to continuously search for neighboring cells, obtain synchronization, and estimate the reception quality of the cell signals, so as to decide whether or not to perform cell search / discovery Re-selection (cell re-selection when the UE is in the RRC_IDLE state), or handover (when the UE is in the RRC_CONNECTUEED state).

Under small cell intensive deployments (as shown in Figure 1), it has been assumed that based on existing standards all small cells within a small cluster (10 small cells in a small cluster) are synchronized (with this synchronization, Network-related performance, such as the use of CoMP, dual-link technology to reduce the search window, etc.). This also brings a certain problem, that is synchronous signal is mapped to the same timefrequency resources, making the inter-cell synchronization signal completely collide together, resulting in a significant reduction in the signal to noise ratio of the received signal, the detection performance dropped sharply. It is difficult to achieve the standard of small cell discovery (the existing standard does not specify the number of small cells found, but at least three). 


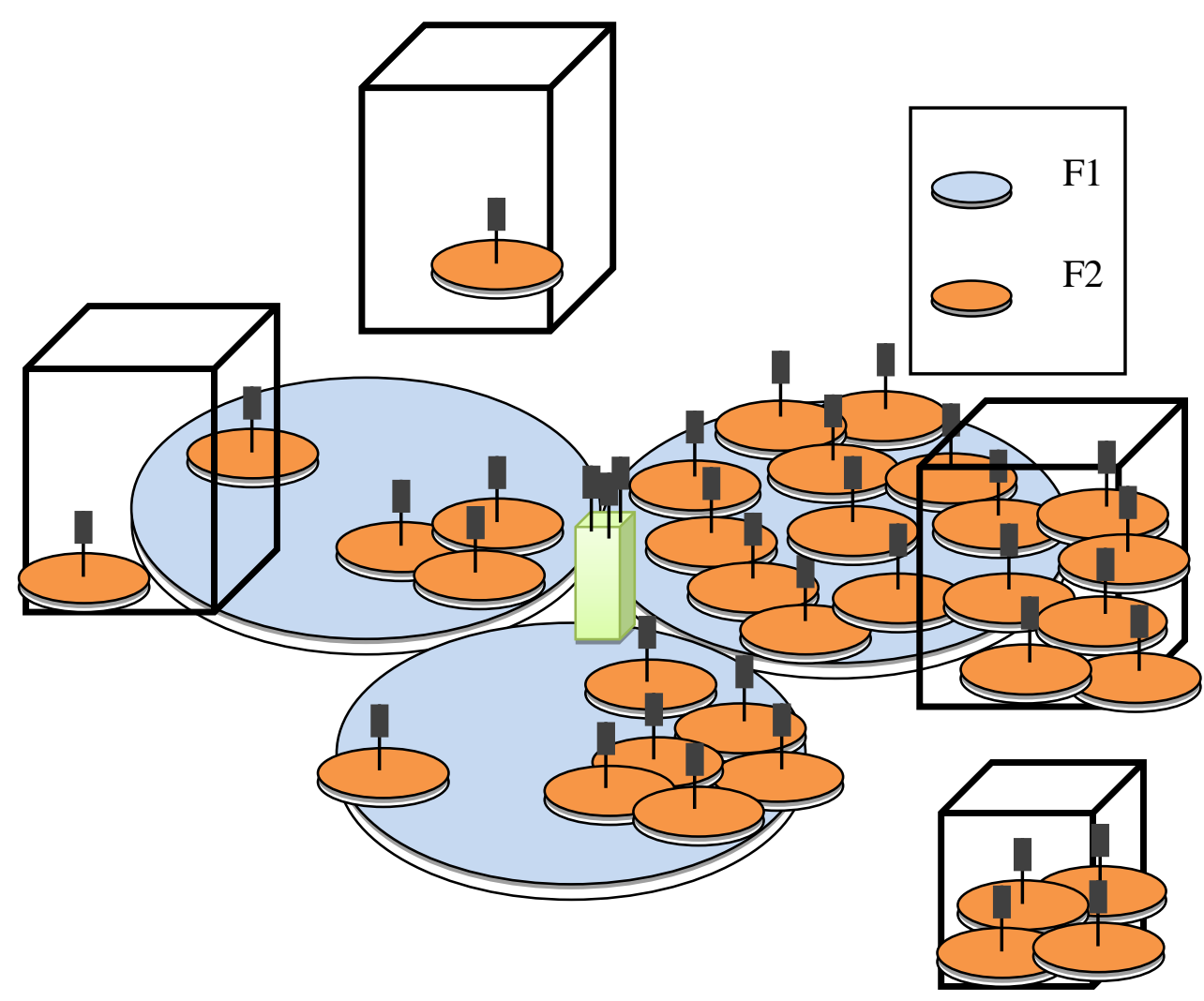

Figure 1. Small Cell Typical Deployment Scenario

\subsection{PSS / SSS under the R8 Mechanisms}

Since the PSS/SSS [3] is transmitted every $5 \mathrm{~ms}$, the average PSS and SSS signal-tonoise ratio is detected by averaging 2 symbols. The small cell is found to be at least $20 \mathrm{~ms}$. When the SINR is less than $0 \mathrm{~dB}$, a longer search time to improve the detection performance of small cells, this will increase the power consumption of UE, especially in the deployment of the network using different frequency search cell.

In the noise-limited scenario (assuming that only the target cell transmits $\mathrm{PSS} / \mathrm{SSS} / \mathrm{CRS}$, interference from other cells is regarded as Gaussian noise), the UE can improve the SINR of the synchronization signal by multiplying the accumulated PSS/SSS. However, in the small cell intensive deployment scenario, the synchronization signals between the cells in the small cell cluster collide completely, as shown in Figure 2, which makes the strong interference between small cells, through multi-frame accumulation and cannot improve the SINR well. The main reason is that PSS/SSS has $10 \mathrm{~ms}$ periodicity, and the cross correlation between PSS/SSS in each cell does not change with time. Within a transmission period of $10 \mathrm{~ms}$, randomization of the interference is only achieved by the SSS signal on subframes 0 and 5. Thus, even with PSS/SSS infinite accumulation, intercell interference cannot be randomized.

In order to reduce the implementation complexity of the synchronization module, the received signal should be down-sampled. Since the primary synchronization signal and the secondary synchronization signal have only 31 subcarriers on both sides of the DC subcarrier DC, the bandwidth of the digital baseband filter Just for the 31 sub-carrier bandwidth, so that the synchronization signal can be filtered out to remove the other subcarrier signal on the synchronization signal interference. 


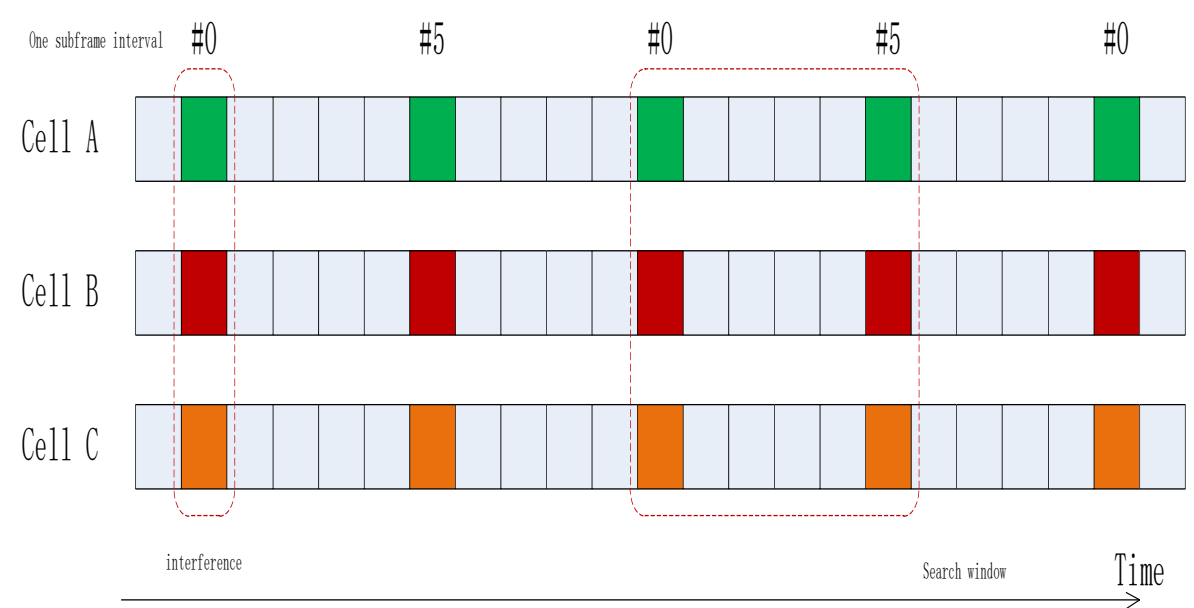

Figure 2. Cell Search Under Synchronization Network (Synchronization Signal does not Change with Time)

\subsection{PSS/SSS Interval Transmission (PSS / SSS Muting)}

The small cell discovery technique using PSS / SSS can not effectively utilize the small cell signal which has been found in the small cell detection, which makes it very strong interference every time when searching small cell. Small sub-district and weak small area, the interference from strong small area and other small area can not be neglected, which makes it difficult to detect the effective small area. The small cell detection technique based on PSS / SSS interference cancellation effectively utilizes the detected small cell signals. When detecting sub-strong cells and weak cells, the received signals are reconstructed to remove the small cell signals that have been detected, The interference from the detected small-cell signals is reduced. The basic idea is to detect the signal layer by layer, each detection error probability of the smallest signal, and then the received signal as interference, from the received signal to eliminate its impact on the residual signal.

Based on the traditional cell search mechanism, the UE can detect the PSS/SSS signal in the center band 6PRBs with the unknown system bandwidth, and establish the timefrequency synchronization with the target cell. PSS/SSS of all cells interfere with each other when the small cell is deployed in the synchronous network.

In order to reduce PSS/SSS interference between the cells and improve the SINR of the received signal, PSS/SSS interval transmission can be applied [4]. As shown in Figure 3, the cells 0,1 , and 2 transmit the synchronization signal in turn (the cells with the same PSS root sequence number may simultaneously transmit the synchronization signal). In order to achieve better detection performance, the auxiliary information may be provided to the UE in an interval transmission mode specified by the target cell. If the UE does not know the information of the interval transmission mode, the UE receiver may process the signal of the interval transmission position as the noise interference process, affect its detection performance improvement is limited. For the PSS/SSS signals under the traditional cell, the interval transmission can be completely controlled by the network layer for backward compatibility. 


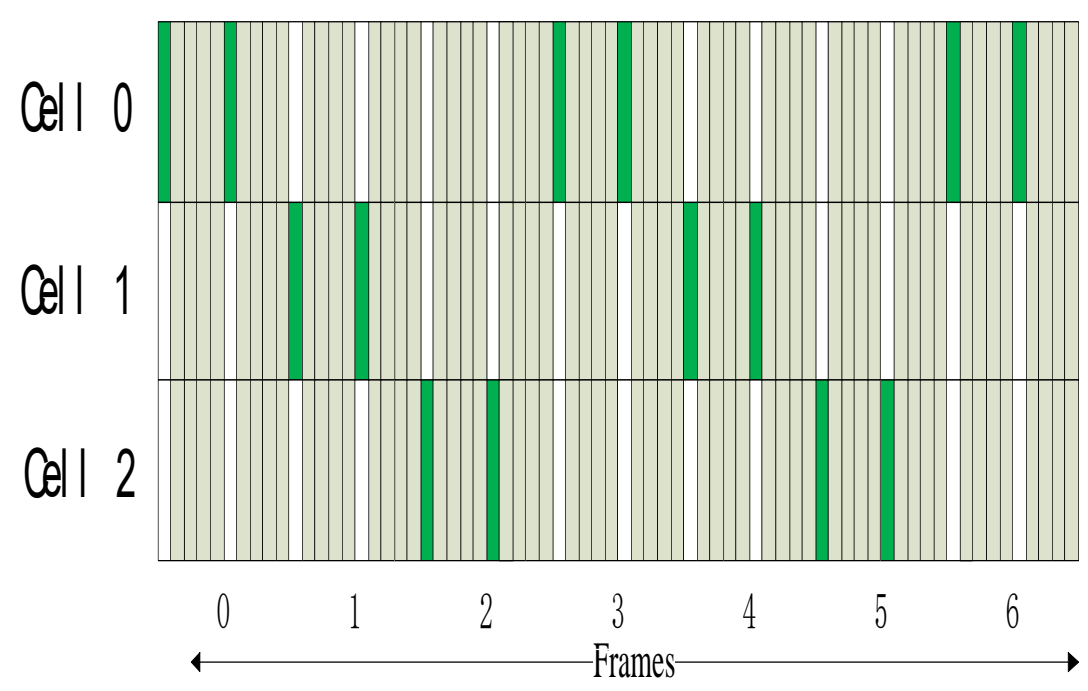

Figure 3. PSS / SSS Interval Transmission

PSS/SSS interval transmission mode can provide the same level of existing PSS/SSS cell search function, and the detection performance can be significantly improved. The cell search function based on the R8 mechanism can be reused when the auxiliary information provides the relevant interval transmission mode information to the UE. This model only requires high-level definition of the corresponding interval mode, the existing standard has little effect, but its performance can be greatly improved.

In the small cell discovery technique based on the original PSS / SSS interference cancellation algorithm, when the strong small cell is detected, the interference is mainly from the small and weak sub-cells, which has little influence on the detection of stronger small cells. The strongest small-area signal will be eliminated, and the strongest smallarea signal will be eliminated. In the process of detecting the strong small-cell, it is necessary to reconstruct the received signal, Which will affect the reconstructed substrong cell signal detection and its channel estimation. In the same way, when weaker cells, because of the previous two channel estimation when there is a certain error, which will affect the weaker small cell signal reconstruction and detection.

\subsection{PSS / SSS Interference Cancellation}

PSS/SSS interference cancellation has been introduced in R11; PSS/SSS IC [5] specific programs are as follows:

(1) UE performs a cell search function (including time and frequency synchronization and the target cell ID) to search for a potential strongest cell;

(2) UE performs channel estimation on the cell searched in step (1);

(3) UE removes the cells obtained after the above two steps.

For potential sub-strong cells, it should repeat the above steps for iteration. The above steps (2) and (3) are not performed in the cell search procedure of R8/R9/R10. In addition, the above steps (2) and (3) to bring additional complexity to the UE. The number of iterations (the number of cells required to search) will also affect the UE power consumption. According to the existing standards, at least three cells are searched and eliminated correspondingly, which greatly affects the complexity and power consumption of the UE. Therefore, the overall performance and complexity of the PSS/SSS interference cancellation is determined by the number of cells detected by the UE ability. 
It can provide good detection performance if UE power consumption is not taken into account.

\subsection{Other RS Detection Mechanisms}

3GPP discusses the characteristics of discovery signal (DS), which is generally believed that the DS should be able to improve the performance of the receiver compared to the existing downlink reference signal, in view of the above mentioned problem of excessive power consumption and high complexity of the UE SINR of the signal, and the UE can discover a plurality of small cells at a time or at a small number of attempts, thereby reducing the detection time. In order to achieve the above-related functions, DS should meet the following characteristics:

(1) The density of the DS in one subframe is much higher than that of the existing downlink synchronization signal;

(2) One or a few DS transmission cycle is longer;

(3) DS should support a sufficient number of orthogonal sequences and quasiorthogonal sequences, to reduce inter-cell interference.

In addition, in order to improve the UE energy efficiency in discovering small cells, the macro base station can provide relevant auxiliary information to the UE (high layer signaling support is required), and the DS of all the small cells should be transmitted synchronously, as shown in Figure 4, Search window to reduce the UE power consumption.

When the non-interference cancellation algorithm is adopted, the number of different measurement samples has little impact on the detection probability of the weak small cell C, only $20 \%$ can be achieved, mainly because the strong small cell A and the sub-strong cell $\mathrm{B}$ and other small cell Interference. When using the interference cancellation algorithm, the detection probability is greatly improved compared with the noninterference cancellation algorithm, and the detection probability is $64 \%$ in the 10 sets of measurement samples. Using the improved PSS / SSS interference cancellation algorithm Which is $91 \%$ because the improved algorithm mainly improves the channel estimation accuracy of the strong small cell $\mathrm{A}$ and the sub-strong small cell $\mathrm{B}$. The original iterative error is reduced and the reconstructed weak small cell $\mathrm{C}$ Of the signal is more accurate, then the detection probability has been greatly improved.

This chapter mainly introduces the flow and typical deployment scenario of Small Cell discovery technology, and introduces the small cell discovery technology based on PSS / SSS. Since it does not use the detected small cell signals when detecting small cells, By a strong interference, detection capacity is low. The small cell detection technique based on the PSS / SSS interference cancellation algorithm can effectively utilize the detected small cell signal, but it has the error propagation problem. The small cell signal precision first affects the reconstruction of the small cell signal, which leads to its low probability of detecting weak small cells. The improved algorithm is mainly aimed at the above problems, and the corresponding improvement is made on the basis of the original process. When the small strong cells are detected, the received signals are reconstructed again, redetection of strong small area, this time a smaller area of interference by a certain reduction, and then a strong small cell signal elimination, detection of small strong subdistrict, and finally weaker small area, through the final simulation The results can also be verified, the detection performance has been improved. 


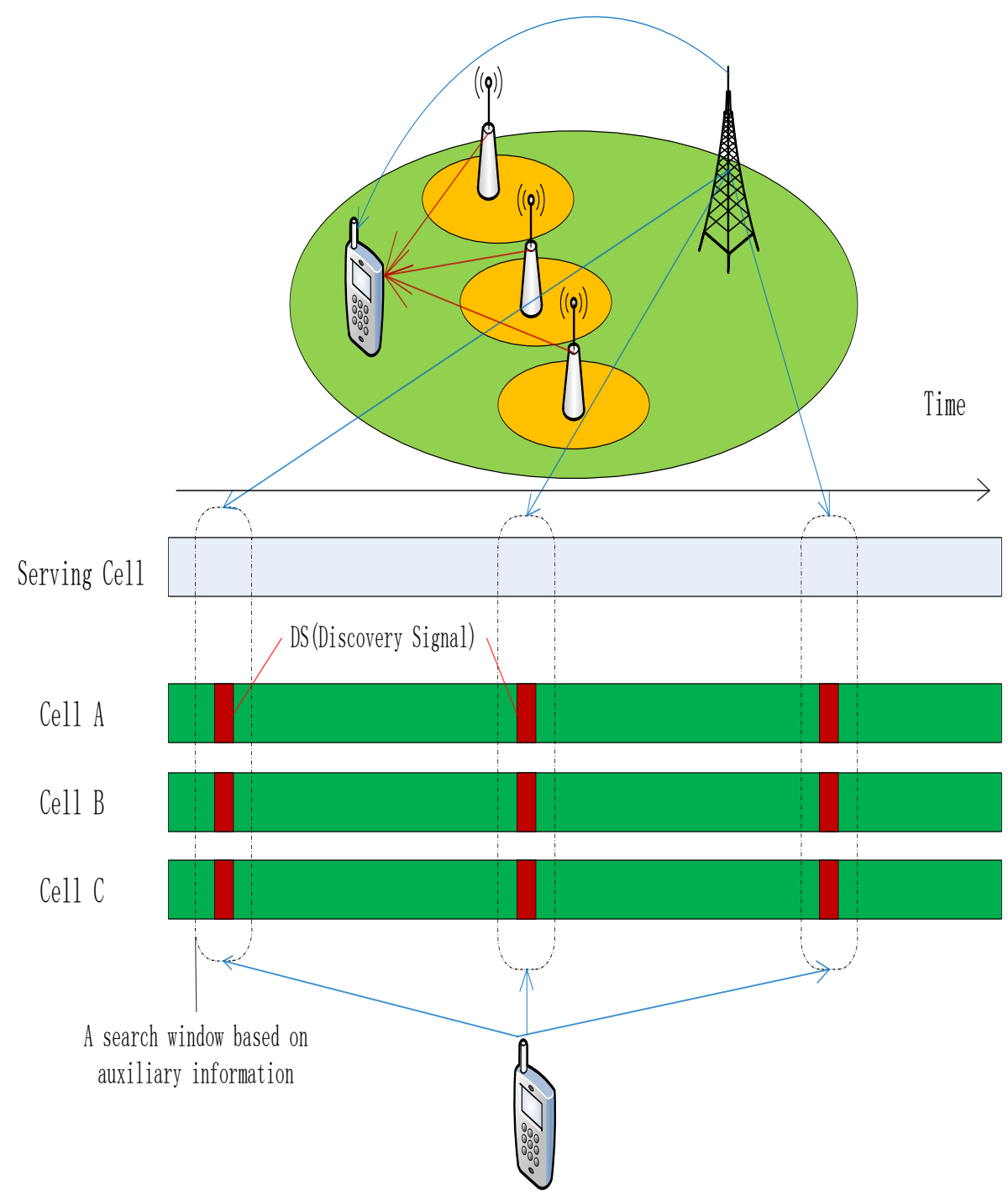

Figure 4. Based on Network Synchronization and Auxiliary Information of the Cell Discovery Mechanism

The existing downlink reference signals CRS, PRS and CSI-RS [6] can be considered as the DS for the relevant requirements of the above DS signal design. As shown in Figure 5, each small cell in a small cell cluster tries to transmit a different reference signal pattern CRS/PRS/CSIRS, in order to reduce the interference, in the case of CRS, PRS or CSI-RS, The resources used by the CRS/PRS/CSI-RS transmitted by the small cell should be set to 0 in the other small cell so that all the DS in a small cell cluster are orthogonal. 

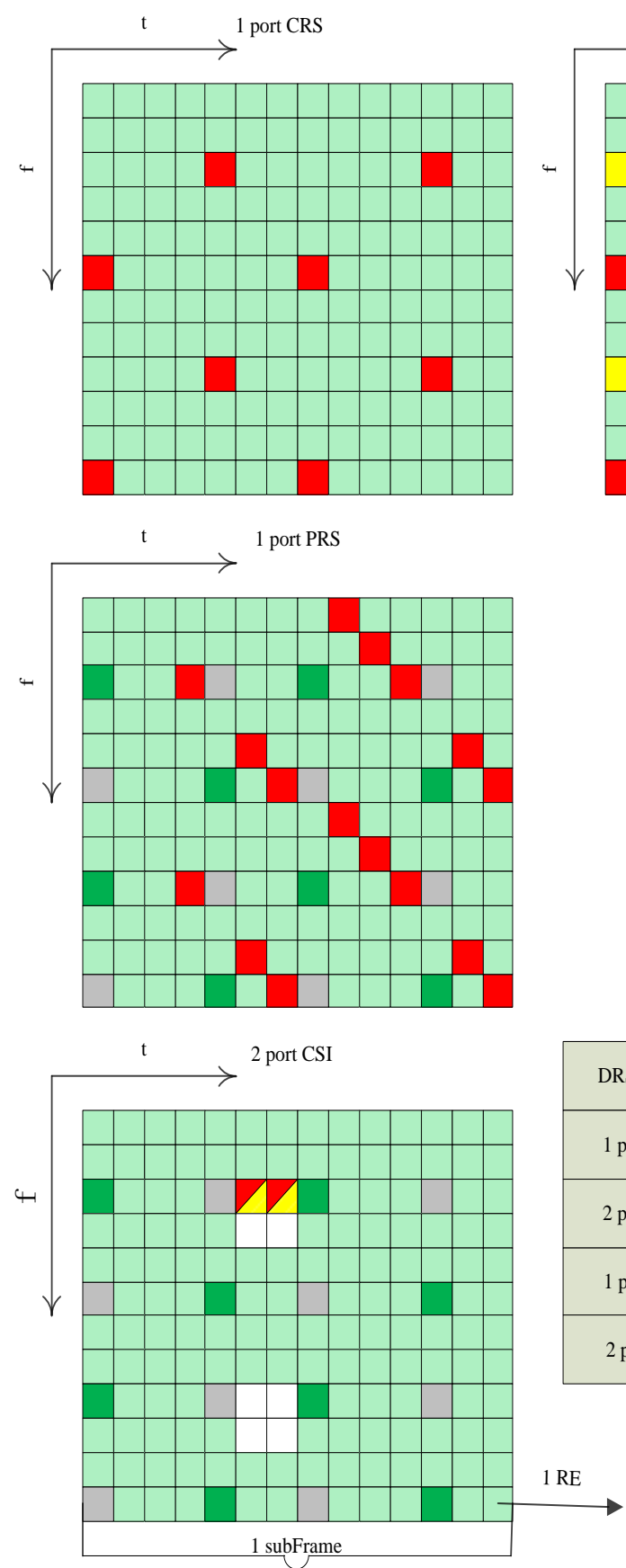

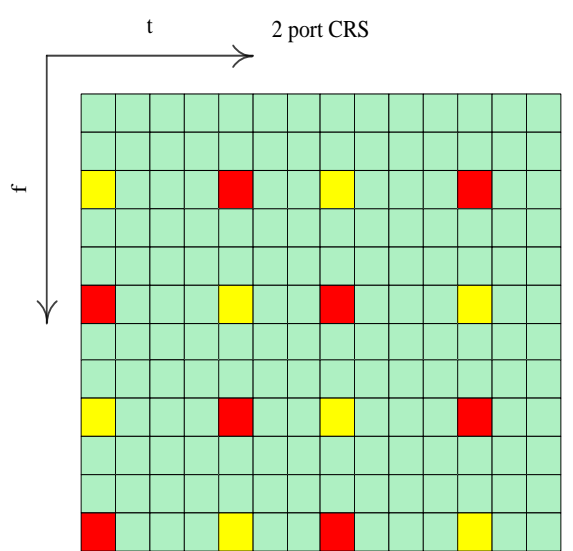

Data

CRS port \#0

CRS port \#1

DRS port \#0

DRS port \#1

Blank

\section{Figure 5. Possible Signal Design Based on the Presence of the LTE Reference Signal}

PRS or CSI-RS as DS may choose to occupy different numbers of PRBs in the frame, may be $6,25,50$ PRBs, the specific configuration of which may be communicated to the UE by the macro base station, but requires all small cells in a small cell cluster The PRS/CSI-RS occupies the same number of PRBs.

In order to speed up the performance of the UE discovery cell, LPP [7] defines the relevant auxiliary information (physical layer cell ID, antenna port configuration, CP type, PRS bandwidth, PRS configuration, UE receiving PRS time provided by PRS as DS. etc.) to choose from PSS/SSS occupies $62 \times 2 \times 2=248$ REs, even if PSS/SSS uses 2 samples, occupying 62 REs in each subframe, as compared with the conventional synchronization 
signal in small cell discovery. Normal CP, 1 or 2 antenna ports, assuming that the PRS is transmitted on $50 \mathrm{PRBs}$, the PRS occupies $50 \times 16=800 \mathrm{REs}$, compared to the detected REs, $800 / 248=3.2$, which means that the PRS can make the UE get better detection performance.

This mechanism requires high level signaling to accelerate the efficient operation of the small cell mechanism. It does not provide backward compatibility, but it can provide good detection performance and reduce the UE search time and power consumption.

\subsection{Comparison of Four Discovery Mechanisms}

Through the above analysis, the traditional mechanism of PSS/SSS found need a long time in the district, UE power consumption is high, by the way the cumulative frame and cannot improve its detection performance; The power consumption of the PSS/SSS interference cancellation algorithm depends on the number of cells it detects, when the number of cells is 3, the power consumption is high; The PSS/SSS interval transmission performance is the best, the impact of the existing norms and the UE is less, 3GPP also focused on the PSS/SSS interval transmission mode ; PRS/CSI-RS can also achieve good discovery performance if the upper layer can provide corresponding auxiliary information, and its detection time is shorter and power consumption is smaller. Specific comparison shown in Table 1:

Table 1. Comparison of Four Discovery Mechanisms

\begin{tabular}{|l|l|l|l|l|}
\hline & \multicolumn{1}{|c|}{ PSS/SSS } & \multicolumn{1}{|c|}{$\begin{array}{c}\text { PSS/SSS } \\
\text { muting }\end{array}$} & PSS/SSS IC & PRS \\
\hline Time synchronization & Able & Able & Able & Able \\
\hline $\begin{array}{l}\text { UE complexity and } \\
\text { power consumption }\end{array}$ & High & Low & High & Low \\
\hline Special effects & None & Small & Small & Large \\
\hline Detection performance & Low & High & High & High \\
\hline
\end{tabular}

\section{Conclusions}

Small cell as an emerging technology in the R12 standard occupies an important position. 3GPP R12 stage of the small cell deployment scenarios, physical layer enhancements, high-level enhanced functions were studied. Small cell discovery technology to solve the small cell in intensive deployment scenarios, the user found small cell mechanism and technical solutions. In this paper, several potential schemes of small cell discovery technology are analyzed in detail, and their influence on the existing standards and their backward compatibility are also analyzed. The power consumption and complexity of UE are also considered. And its future research directions are also discussed.

As the SINR of different small interval signals is improved, the detection performance is improved obviously. It can be seen that the detection performance of the randomized grouping scheme is significantly improved compared to the conventional scheme, especially for the weaker Small Cell because the small inter-cell interference is very severe in the conventional scheme, and in the random grouping scheme, Interference has been a certain ease. The performance improvement can be further improved by comparing the improved scheme with the stochastic grouping scheme, because it takes into account the interferences between the small zones well and further reduces the interference between the small zones, and the detection probability is improved accordingly. 


\section{References}

[1] S. F. Chou, T. C. Chiu and Y. J. Yu, "Mobile small cell deployment for next generation cellular networks", 2014 IEEE Global Communications Conference (GLOBECOM). Austin, TX: IEEE Press, (2014), pp. 4852-4857.

[2] G. K. Chang, L. Cheng and Z. Liang, "Architecture and applications of a versatile small-cell, multiservice cloud radio access network using radio-over-fiber technologies", 2013 IEEE International Conference on Communications Workshops (ICC). Budapest: IEEE Press, (2013), pp. 879-883.

[3] T. Yamamoto and S. Konishi, Impact of small cell deployments on mobility performance in LTEAdvanced systems", 2013 IEEE 24th International Symposium on Personal, Indoor and Mobile Radio Communications (PIMRC Workshops), London: IEEE Press, (2013), pp. 189-193.

[4] 3GPP TS 36.213 V12.4.0. 3rd Generation Partnership Project; Technical Specification Group Radio Access Network; Evolved Universal Terrestrial Radio Access (E-UTRA); Physical layer procedures(Release 12) [S], (2014).

[5] 3GPP TS 36.211 V12.4.0. 3rd Generation Partnership Project; Technical Specification Group Radio Access Network; Evolved Universal Terrestrial Radio Access (E-UTRA); Physical channels and modulation (Release 12) [S], (2014).

[6] T. Ohseki, T. Yamamoto and Y. Suegara, "Performance evaluation of LTE-Advanced downlink adopting higher order modulation in small cells", 2015 IEEE Radio and Wireless Symposium (RWS). San Diego, CA: IEEE Press, (2015), pp. 120-122.

[7] 3GPP TS 36.104 V12.6.0. 3rd Generation Partnership Project; Technical Specification Group Radio Access Network; Evolved universal terrestrial radio access(E-UTRA); Base Station(BS) radio transmissin and reception(Release 12) [S], (2014).

\section{Authors}

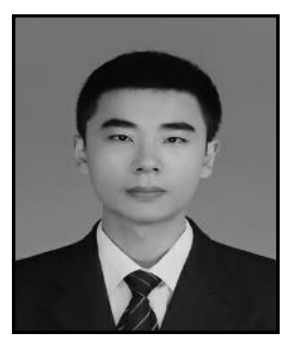

Youqi Shen, got his B.S. degree from Weifang University, and his Master degree from Chongqing University of Posts and Telecommunications, year in 2014 and now. His main research is mobile communication terminal technology. 